\title{
AMMONIA BLOOD LEVELS AND VALPROIC ACID
}

The prevalence and magnitude of valproic acid (VPA)-related hyperammonemia (HA) were estimated by a review of the literature, and the clinical symptomatology and risk factors for the development of HA determined by researchers at university hospitals in Santiago, Chile; and Rush University Medical Center, Chicago, IL. Out of 183 studies between 1980 and 2005, 24 met inclusion criteria; 7 were prospective and 17 cross-sectional. The prevalence of $\mathrm{HA}$ in prospective studies ranged from $70 \%$ to $100 \%$ (mean $90.3 \%$ ), and in cross-sectional studies it varied between $16 \%$ and $100 \%$ (mean $47.4 \%$ ). Ammonia blood levels increased two-fold on average compared to baseline. In one study, children younger than 2 years treated with VPA to a plasma level above $100 \mathrm{mg} / \mathrm{L}$ were at greater risk of developing HA; 4 studies found no significant correlation between age and HA. Two studies in children found a relation between VPA levels and HA. HA was not related to clinical symptoms. Hepatic dysfunction was not observed in 11 studies with liver function tests. HA correlated with administration of other antiepileptic drugs and polytherapy. (Chicharro AV, de Marinis AJ, Kanner AM. The measurement of ammonia blood levels in patients taking valproic acid: looking for problems where they do not exist? Epilepsy \& Behav Nov 2007;11(3):361-366). (Respond: Ada Chicharro c/o Andres Kanner, Department of Neurological Sciences, Rush University Medical Center, Epilepsy Center, 1653 West Congress Parkway, Chicago, IL 60612).

COMMENT. The administration of concomitant antiepileptic polytherapy was most frequently associated with hyperammonemia (HA). Age, and dose and blood levels of VPA were not significant risk factors for HA, except for children younger than 2 years who may have a greater susceptibility to develop HA. Based on this review, measurement of ammonia blood levels in asymptomatic patients taking VPA is not advised and may lead to diagnostic confusion. HA alone is not an indication to discontinue VPA. Ammonia blood levels are recommended in patients taking VPA who exhibit signs of acute encephalopathy. Alternative causes for an encephalopathy should also be investigated in VPA-treated patients with HA.

\section{ATTENTION DEFICIT DISORDERS}

\section{FREQUENCY AND ETIOLOGY OF ADHD IN NEW ONSET EPILEPSY}

The prevalence, complications and etiology of ADHD were determined in 75 children (age 8-18 years) with new/recent onset idiopathic epilepsy nd 62 healthy first-degree cousin controls, in a study at University of Wisconsin School of Medicine and Public Health, Madison, WI; and Rosalind Franklin University of Medicine and Science, North Chicago, IL. ADHD was present in $31.5 \%$ of children with new onset epilepsy compared to $6.4 \%$ of healthy controls $(\mathrm{P}<0.001)$. Among children with epilepsy and ADHD, 52.1\% (12/23) had the inattentive subtype, $17.4 \%(4 / 23)$ were hyperactive subtype, $13.1 \%(3 / 23)$ were combined type, and $17.4 \%(4 / 23)$ wsere NOS subtype. ADHD was present before seizure onset in $19 / 23$ children (82\%). The ADHD+ children were more likely to have an IEP than controls (52.2\% vs $15.4 \%, \mathrm{P}<0.001)$, or they had more supportive academic services $(69.6 \%$ vs 
$38.5 \%, \mathrm{P}<0.001)$. Educational resource services to address academic underperformance were provided before epilepsy was diagnosed in $65.2 \%$ of epilepsy ADHD+ children vs $30.8 \%$ of epilepsy ADHD- group $(\mathrm{P}<0.001)$. Neuropsychological impairments in motor/psychomotor speed and executive function were observed in the epilepsy ADHD+ group. No differences were found between the 2 groups in the proportion of children with comorbid depressive disorders, anxiety, tic disorders, or psychotic disorders. ODD was more frequent in ADHD+ group ( $30.4 \%$ vs $2 \%$ comorbidity, $\mathrm{P}<0.001)$. Patients with complications of pregnancy and birth were not more prevalent in the ADHD+ group compared to ADHD- group. Quantitative MRI showed that ADHD in epilepsy is associated with significantly increased gray matter in regions of the frontal lobe and significantly smaller brainstem volume. No differences were observed between the ADHD+/- groups in terms of epilepsy syndrome, treatment with AED, or age of onset or duration of epilepsy. The etiology of ADHD in children with epilepsy is not related to recurrence of seizures and their treatment. Neurodevelopmental abnormalities involving the frontal lobe gray matter antedate the onset of seizures and contribute to the etiology of ADHD and associated comorbidities. (Hermann B, Jones J, Dabbs K, et al. The frequency, complications and etiology of ADHD in new onset pediatric epilepsy. Brain December 2007;130:3135-3148). (Respond: Dr Bruce Hermann, Matthews Neuropsychology Lab, Department of Neurology, University of Wisconsin, Madison, WI 53792).

COMMENT. ADHD, especially the inattentive subtype, is a common comorbid disorder in children with epilepsy, and it antedates the onset of seizures, according to the above report. The etiology of ADHD in epilepsy is not related to the frequency or type of seizures or their treatment. The authors confirm previous findings of frontal lobe neurodevelopmental abnormalities as underlying factors in the etiology of ADHD.

In a proposed etiological classification of ADHD, a genetic factor is estimated in approximately $80 \%$ of cases, involving dopamine receptor and dopamine transporter genes.. Developmental cerebral abnormalities and environmental factors are contributory (Millichap JG. Pediatrics Feb 2008;121(2):in press). These are classed as prenatal, perinatal, and postnatal. They involve pregnancy and birth-related risk factors, including maternal smoking and alcohol ingestion, prematurity, HIE, and thyroid deficiency. Childhood illnesses associated with ADHD, in addition to epilepsy, include virus infections, meningitis, encephalitis, head injury, toxins, and drugs. Iron and zinc deficiencies, and diet-related sensitivities are more controversial factors. Febrile seizures, frequently associated wih HHV6 and influenza A, are a risk factor for subsequent development of hyperactive behavior and ADHD. A greater emphasis on etiology might encourage a preventive or specific approach to treatment, with less reliance on symptom modification with medications.

Cardiac safety of CNS stimulants in ADHD. A retrospective cohort study of 3 to 20 year old patients newly diagnosed with ADHD found no cardiac death during 42,612 personyears of stimulant use. Hospital admissions for cardiac cause occurred for 8 children during stimulant use, and 11 during former use. Current stimulant use was associated with a $20 \%$ increase in emergency department cardiac visits when compared with nonuse. Former use was not a risk factor. Reasons for ED cardiac visits included syncope, dysrhythmia, tachycardia, and hypertension. None was identified as addiction to stimulants. (Winterstein AG, Gerhard T, Shuster J, et al. Cardiac safety of central nervous system stimulants in children and adolescents with attention-deficit/hyperactivity disorder. Pediatrics December 2007;120(6):e1494-e1501). (Respond: AG Winterstein PhD: E-mail: almut@cop.ufl.edu). 